\title{
Evidence of means-end behavior in Asian elephants (Elephas maximus)
}

\author{
Naoko Irie-Sugimoto $\cdot$ Tessei Kobayashi $\cdot$ Takao Sato $\cdot$ \\ Toshikazu Hasegawa
}

Received: 23 April 2007 / Revised: 16 November 2007 / Accepted: 2 December 2007 / Published online: 18 December 2007

(C) The Author(s) 2007

\begin{abstract}
The present study explores to what extent Asian elephants show "means-end" behavior. We used captive Asian elephants $(N=2)$ to conduct four variations of the Piagetian "support" problem, which involves a goal object that is out of reach, but rests on a support within reach. In the first condition, elephants were simultaneously presented with two identical trays serving as the "support", with the bait on one tray and the other tray left empty. In the next two conditions, the bait was placed on one tray, while additional bait was placed beside the other tray. In the last condition, both trays contained bait, but one of the trays had a small gap which prevented the elephants from reaching the reward. Subjects were required to choose and pull either tray with their trunk and to obtain the bait (i.e. goal). Results showed that one elephant performed all of the support problems significantly above chance after several sessions, suggesting that the elephant was capable of understanding that pulling the tray was the "means" for achieving the "end" of obtaining the bait. This study showed that elephants show means-end behavior when
\end{abstract}

\footnotetext{
N. Irie-Sugimoto $\cdot$ T. Sato

Department of Psychology,

Graduate School of Humanities and Sociology,

University of Tokyo, Tokyo, Japan

N. Irie-Sugimoto $(\varangle) \cdot$ T. Kobayashi $\cdot$ T. Hasegawa

Department of Cognitive and Behavioral Science,

Graduate School of Arts and Science,

University of Tokyo, Tokyo, Japan

e-mail: naokois@darwin.c.u-tokyo.ac.jp

Present Address:

T. Kobayashi

NTT Communication Science Laboratories,

NTT Corporation, Kyoto, Japan
}

subjected to a Piagetian "support" task, and indicates that such goal-directed behavior occurs in species other than primates.

Keywords Asian elephant · Piaget · Means-end problem

\section{Introduction}

Elephants are widely believed to be highly intelligent. Across several countries, elephants have been trained and tamed for public show and labor. Furthermore, elephants easily learn to understand various sorts of commands that are verbally expressed by circus trainers and jungle dwellers (e.g. Rensch 1956, 1957).

Although there is much anecdotal evidence that elephants appear to be intelligent, empirical investigations are scarce. One seminal study examined to what extent Asian elephants possess the ability to memorize sets of arbitrary drawings (Rensch 1957). In that study a young female elephant was presented with a pair of drawings and then required to choose the drawing that had been arbitrarily predetermined to be the "correct" one. After several training sessions (330 trials in total), she had learnt to choose the correct drawing. When different pairs of drawings were presented, the elephant learned the discrimination more and more rapidly (within 10 trials by the fourth pair), and could select them even after 1 year. The excellent memory performance of elephants was also described in a different study. McComb et al. (2000) showed that an African elephant recognized the low-frequency contact calls of herd members who had died 23 months before, and even of individual who had left the herd 12 years earlier.

Other evidence of elephant intelligence is their capacity to use tools skillfully. Hart et al. (2001) observed that wild 
and captive Asian elephants use sticks as tools to fend off flies and to scratch their own bodies (e.g. Hart and Hart 1994). Moreover, they reported that elephants sometimes modify sticks by breaking them in half when they are too long or by taking leaves off branches when they are too leafy (Hart and Hart 1994). Because it was long believed that only a few species such as chimpanzees and crows spontaneously use tools in the wild (e.g. Gould and Gould 1994; Vauclair 1996), the elephants' ability to use tools seems intriguing in terms of theoretical arguments about animal intelligence.

As noted above, elephants appear to possess high-level cognitive abilities in various situations. Their intelligence potential is also supported by their anatomical and physiological features. Elephants, especially Asian elephants (Elephas maximus), have the largest brain of all terrestrial species (approximately 5,000 g). Moreover, their encephalization quotient (EQ), i.e. the ratio of cortex to body size, is high (2.30, compared to 2.49 for chimpanzees and 7.44 for humans, e.g. Jerison (1974)). According to theoretical arguments, such a large brain is likely related to high cognitive performance (Jerison 1974). However, Nissani (2006) recently reported the poor performance of elephants when given a causal reasoning task. In the task, the elephants were to simply remove the lid off a bucket to earn the bait inside, but some elephants still removed the lid when the lid was placed on the ground therefore unrelated to the goal of obtaining the bait inside the bucket. Author concluded elephants did not form a causal model, in which the lid is perceived as an obstacle to food underneath it. However, as the author himself points out, it is possible that the results speak more to the rigidity of elephants' learned behavior to their lack of causal reasoning. Hart et al. (2007) argue that less-densely packed and larger elephants' cortical nonsomatic neurons may give an account of their better performance in long-term extensive memory but rather poor performance in conventional test of cognitive performance such as causal reasoning tasks.

Furthermore, elephants are unique in terms of possessing a prehensile trunk. The trunk makes it possible to manipulate easily various types of objects, even objects as small as a straw from a floor. Thus, in some way an elephant's trunk may be comparable to the skillful hands of primates.

In the present study, we examined whether Asian elephants show means-end behavior. We used a Piagetian "support" task, in which this specific kind of problem-solving skill is assessed. Piaget (1952) first used the support task to investigate whether human infants are able to understand means-end relationships. He developed a task in which the infant must try to obtain a toy that is out of direct reach by pulling a towel placed underneath the toy toward itself. In this task, the towel serves as a support and applying the support in the correct way is the means to accomplish the end of obtaining the toy. Human infants of around
11 months of age have acquired the ability to complete the task, which indicates that they possess conceptual knowledge about the relationship between the means and the end, the spatial relationship between the two, and the features of the material serving as a support. Moreover, it indicates the development of cognitive processing that allows subjects to have a definite goal, to persist in attempting to achieve it, and to produce a goal-directed behavior. Similar cognitive steps are required for tool-use behavior. Thus, this task may also serve to assess tool-using ability. Subsequent studies have developed the support task to examine in more detail the development of means-end understanding in early infancy (e.g. Willatts 1984, 1990, 1999).

Previous studies have reported that nonhuman primates such as New World monkeys and chimpanzees are capable of understanding a means-end relationship in a variety of experimental tasks (Hauser et al. 2002, 1999; Povinelli et al. 2000). Cotton-top tamarins, for example, have been shown to perform the support task well in a laboratory setting (Hauser et al. 2002). Tamarins were to choose between a board on which bait was placed and thus served as a support when drawn closer and the other board to which bait was placed next and thus would not serve as a support. Hauser et al. (1999) also tested whether the tamarins would generalize to changes in the irrelevant features of the support, such as the shape, size, and color, which they succeeded. Moreover, the tamarins were able to reach the criterion (attain an accuracy of $90 \%$ on two consecutive sessions) within 260 trials in the on-off problem tasks (Hauser et al. 2002). In another study with tamarins, they were able to learn to earn baits involving two tools in combination, though they do so only with sufficient training (Santos et al. 2005).

Other studies had investigated the ability in other mammals and birds. For instance, Halsey et al. (2006) showed wild common marmosets were able to solve the parallel string task, in which one of the two strings was attached to bait, and subjects were to select the attached string to earn the bait, under natural condition. Osthaus et al. (2005) showed that unlike apes, dogs are able to learn to pull on a string to earn the attached bait but they do not solve the task by virtue of understanding the means-end relationship. Pigeons were able to learn the discrimination of two ribbon/ dish assemblies, in which pulling the ribbon allowed pigeons to retrieve the dish full of baits (Schmidt and Cook 2006). However, their results did not indicate the evidence of comprehension of conceptual nature of connectedness. In another study, Heinrich and Bugnyar (2005) showed that ravens were able to pull on a string to retrieve the attached meat, and they did this without initial training.

In the present study we investigated the cognitive ability of Asian elephants by testing their capacity for showing means-end behavior when subjected to a Piagetian "support" task. They were given a means-end task, modeled 
after the Hauser et al.'s (1999) study on cotton-top tamarins, and were given two alternatives of identical boards, in which one of them would serve as a support to bring baits into their reach.

\section{Methods}

Subjects and housing

Data were collected from two female captive Asian elephants (E. maximus), Authai (aged 6 years) and Surya (aged 10 years), which were born in Thailand and India, respectively. They were raised by humans in peer groups during infancy and then moved into the Ueno Zoological Park in Tokyo, Japan. They were housed together for 3 years in adjacent home cages $(6.5 \mathrm{~m} \times 6 \mathrm{~m})$ with an older female ( 27 years old). Each home cage was connected to a larger outside enclosure (approximately $35 \mathrm{~m} \times 40 \mathrm{~m}$ ), where the elephants played together in the daytime. Thus, they had considerable physical, vocal, and tactile contact with conspecifics. All facilities were approved by the Japanese Association of Zoos and Aquariums, and the housing was based on the Guidelines for the Management of Asian Elephants in Japan.

The elephants were managed in a free-contact way, ensuring that a zookeeper had free access to the enclosure, managed them, kept their bodies clean, and sometimes trained them to obey some types of vocal command ("go", "wait", "sit down", etc.). However, they were not trained to perform in show for the public. The elephants were fed four times a day, 8:00, 11:00, 13:00 and 16:00, on a diet of hay, soilage, and fruits (sometimes a vitamin supplement). They were taken outside before the first meal (8:00), and into the home cage before the last meal (16:00). They had free access to water at all times.

A series of experiments were carried out in an outside playground (as described below in detail) before the second meal (11:00) and/or the third meal (13:00), but not on rainy days, when the elephants would remain inside the home cage all day. The elephant performing in the task was out of view of the other elephant and the order of testing was randomly selected each day. Weights were not especially maintained for the present experiment. The weights $( \pm S D)$ of Authai and Surya were approximately 1,720 $( \pm 20)$ and $2,400( \pm 40) \mathrm{kg}$, respectively, during the experiments. The elephants had no prior experience with any of the experimental tasks.

\section{Procedure}

Prior to testing, we assessed whether the elephants were able to grab and pull a target tray with the tip of their trunk.
The experimenter stood in front of each subject and placed a cardboard tray $(25 \mathrm{~cm} \times 44 \mathrm{~cm} \times 6 \mathrm{~cm})$ approximately $100-120 \mathrm{~cm}$ away from the enclosure. The tray had an extension $(10 \mathrm{~cm} \times 20 \mathrm{~cm} \times 6 \mathrm{~cm})$ with a handle that could be easily grabbed The subjects spontaneously approached, grabbed, pulled, and played with the tray for several minutes. Following the habituation phase, the elephants were subjected to the experimental trials described below.

The general procedure of the experimental trials was as follows: a zookeeper first brought the elephant to a small outside playground $(6.2 \mathrm{~m} \times 6.5 \mathrm{~m})$ between the home cage and the larger outside enclosure. This playground was surrounded by rocks, so that it was not in view of zoo visitors. Within the playground there was an automatic up-anddown gate (195 cm wide). During testing, the gate was kept open and the left and right sides of the opening were connected with a chain at $1 \mathrm{~m}$ height, preventing the elephants from leaving the playground. However, the elephants could reach underneath the chain with their trunks (Fig. 1).

The experimenter stood in front of the gate on the other side of the chain and placed a set of cardboard trays $(25 \mathrm{~cm} \times 44 \mathrm{~cm} \times 6 \mathrm{~cm})$ approximately $100-120 \mathrm{~cm}$ away from the gate. The two trays were always placed side by side, approximately $50 \mathrm{~cm}$ apart. For each experimental trial, the bait (a piece of apple, banana, or watermelon) was placed on or off the trays (for details see below). A 6-cmhigh ridge surrounded each of the trays to prevent the bait from falling out. Because the bait was never directly within reach of the elephants' trunks, they had to grab and pull the tray toward them in order to obtain the bait.

Each trial was initiated when the zookeeper stopped the subject approximately $3.5 \mathrm{~m}$ away from the gate, facing the trays. The zookeeper then gave the elephant a vocal command (i.e., "go"). As soon as the elephant started to walk

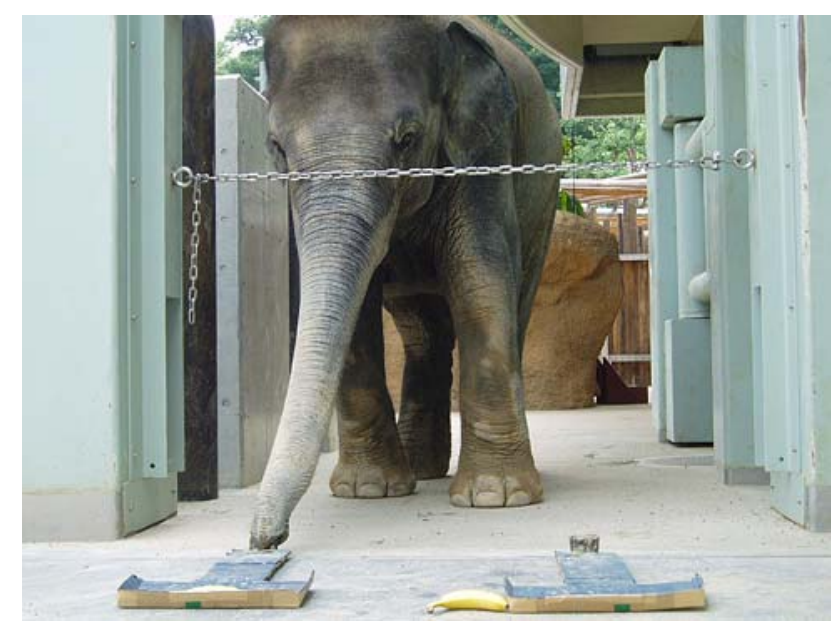

Fig. 1 Surya performing in Condition B. Subjects had to grab the handle and pull the tray closer using their trunk 
toward the tray, the zookeeper turned his back to the elephant to avoid providing any sort of cue. After obtaining the bait, the elephant was pulled away from the gate by the zookeeper. The zookeeper would confiscate the tray from the elephant if she kept grabbing and playing with the tray. While the elephant was away from the gate with the zookeeper, the experimenter recorded the result of previous trial then set up the next trial out of the elephant's sight. The experimenter stood at least $2 \mathrm{~m}$ away from the setting and the elephant facing back and did not face the elephant until the zookeeper pulled the elephant away.

\section{Experimental conditions}

There were four experimental conditions A, B, C, and D. In all conditions the elephants' task was to choose a tray, pull it toward itself, and obtain the bait. A trial was considered "correct" when an elephant chose the tray on which the bait was placed, and retrieved the bait. The correct tray was placed on the right in half of trials and on the left in the other half within a session and the order was random. Both subjects underwent ten successive trials of a single condition on one day (one block).

We first presented the elephants with Condition A, the training condition. Although the significance of performance was predetermined $66 \%$ correct, the elephants were given the same condition trials until their performance met the predetermined criterion, i.e. above $75 \%$ correct in one condition (38/50 trials, binomial test: $P=0.0003$, two-tailed), then the elephant proceeded to the next condition, and so forth, in alphabetical order (A-B-C-D). Detailed materials and procedure of each condition are described below.
Condition A. Ten trials per day were provided to Authai and Surya for 10 and 5 days, respectively. Two identical blue-colored cardboard trays were placed in front of the elephant on the opposite side of the chain. In each trial, the bait was placed randomly on either of the two trays (Fig. 2a). On the first 5 days, the subjects were allowed to pull the trays as many times as necessary until they obtained the bait. On the second 5 days, the subject (only Authai) was allowed to pull the tray only once per trial, and therefore would not obtain the bait if she chose the incorrect tray. The latter procedure was conducted so that Authai would learn that she has only one chance per trial and would not earn bait unless she makes the right choice on her first selection. Surya was not given the second 5-day training sessions because she spontaneously pulled only one tray in her first 5-day training session. The correct performance was defined pulling the baited tray on their first choice for both first and second 5-day training sessions.

Condition $B$. Ten trials per day were provided to Authai and Surya for 15 and 25 days, respectively. The second task is also referred to as the "on-off" task, in which the bait was "on" one tray, and the bait was "off", i.e. lying beside, the other tray (Fig. 2b). Thus, the elephants could access the bait by pulling the tray containing the food, but pulling other tray did not result in such a reward. The trays were the same as those used in Condition A. The subject was allowed to pull the tray only once per trial.

Condition $C$. Ten trials per day were provided to each elephant for 10 days. The third task was a transfer test that can be considered as a variation on the "on-off" task to test whether the elephants are able to make distinction between the relevant and irrelevant features of the problem (Fig. 2c).
Fig. 2 Trays used in Condition A (a), Condition B (b), Condition $\mathrm{C}$ (c), and Condition D (d). Trays were colored blue in Conditions A and B, green in Condition $\mathrm{C}$, and black in Condition D
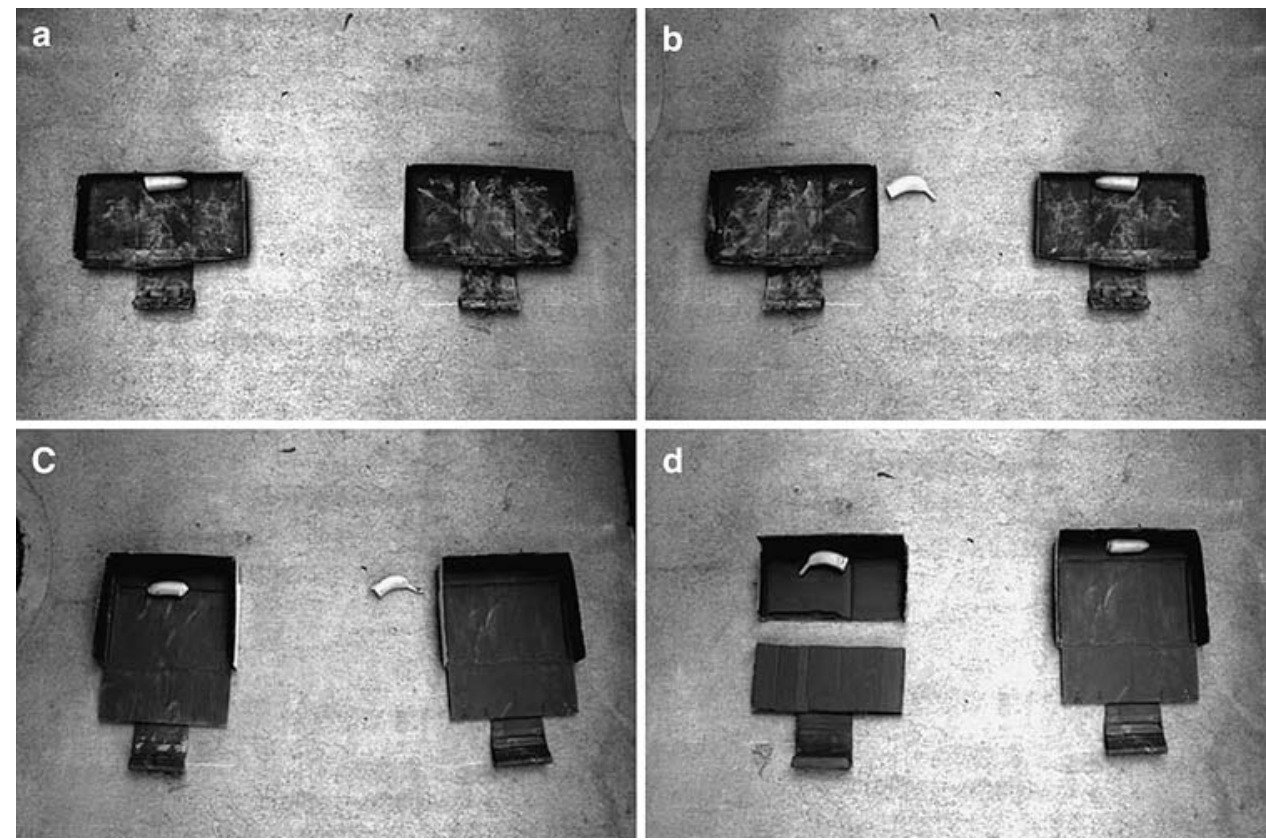
Thus, the task was identical to that of Condition B, except for two irrelevant features: the color of the trays (green instead of blue) and their size $(55 \mathrm{~cm} \times 44 \mathrm{~cm})$.

Condition D. Ten trials per day were provided to Authai for 15 days. This final task is also referred to as the "connectedness" task, in which the elephant had to choose between a bait placed on a single, uninterrupted black-colored tray $(50 \mathrm{~cm} \times 44 \mathrm{~cm})$ and a bait placed on a black-colored tray with a horizontal gap (approximately $15 \mathrm{~cm}$ ) in the middle (lower tray, $15 \mathrm{~cm} \times 44 \mathrm{~cm}$ with a handle; upper tray, $20 \mathrm{~cm} \times 44 \mathrm{~cm})$ (Fig. $2 \mathrm{~d}$ ). The uninterrupted tray was the "correct" choice in this condition, as pulling the other tray would not result in obtaining the bait.

\section{Results}

Figure 3 depicts the percentage of correct trials per block (i.e. 50 trials) across all conditions. Significant performance was set at a score $>66 \%$ correct (33 of 50 trials; binomial test, $P=0.0328)$. The results for the four conditions separately are as follows:

Condition A. Both elephants performed the task significantly better than the chance in the first block (the first 5 days): Authai performed $66 \%$ of trials correctly (binomial test: $P=0.0328$ ), while Surya completed $80 \%$ of trials correctly (binomial test: $P<0.0001$ ) and thus had reached the criterion in the first block. Authai reached the criterion in the second block ( $84 \%$ of trials correct, binomial test: $P<0.0001)$. These findings indicate that the elephants were able to learn that pulling the tray containing the bait enabled them to retrieve the food, whereas pulling the empty tray did not result in such a reward. See Fig. 4 a to see the details of the subjects' early performance.

Condition B. Both subjects required more than two blocks to reach criterion in this task (Authai: $80 \%$ cor-

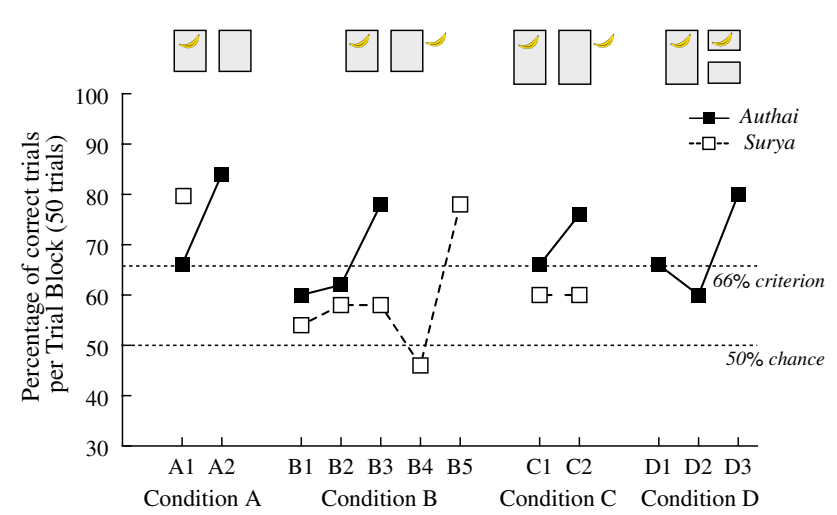

Fig. 3 The percentage of correct trials per block (50 trials). A performance was considered significantly better than chance if $>66 \%$ of trials was correct (33 of 50 trials; binomial test, $P=0.0328$ ) rect at the third block, binomial test, $P<0.0001$; Surya: $80 \%$ correct at the fifth block, binomial test, $P<0.0001)$. These results indicate that the elephants had learnt that pulling the "ON" tray lead to the bait, while pulling the "OFF" tray did not. Authai chose the correct tray in seven trials out of her first ten trials (Fig. 4b).

Condition $C$. Authai performed the task significantly better than chance in the first block $(66 \%$ correct, binomial test: $P=0.0328)$, and reached criterion in the second block ( $78 \%$ correct, binomial test: $P=0.0003)$. Authai chose the correct stimuli in six trials out of her first ten trials. In contrast, although Surya chose the correct stimuli in nine trials out of her first ten trials, Surya performed the task below the significance level in the first and second blocks (60 and 62\% correct, respectively, binomial test: $P>0.1$. See Fig. $4 c)$. The lower performance of Surya might indicate a low level of motivation, and it was decided not to continue the experiments with Surya. Taken together, these findings suggest that one of the two elephants was able to transfer the knowledge obtained under Condition B and apply it to Condition $\mathrm{C}$. The average correct percentage of Authai was $71.1 \%$ and Surya was $61.9 \%$ throughout the task.

Condition $D$. The final task was conducted only by Authai, who scored above chance level in the first block (66\% correct, binomial test: $P=0.0328)$. She chose the correct stimuli in eight trials out of her first ten trials (Fig. 4d). Moreover, she reached criterion in the second block $(80 \%$ correct, binomial test, $P<0.0001)$. These findings suggest that this elephant was able to understand the spatial relationship between the tray and the bait correctly and rapidly, and that she learnt to pull the uninterrupted tray rather than the tray with the gap.

\section{Discussion}

In this study elephants were introduced to tasks from two different categories; first, in the "on-off" task, subjects had to understand the spatial relationship between the tray and the bait. The food had to be on and connected to the surface of the tray for the tray to serve as an effective tool to draw food within the subjects' reach. Second, to solve the "connectedness" problem, subjects had to learn that the food had to be on and connected to the surface of a single uninterrupted tray instead of on a tray with a horizontal gap, thus making this problem more complicated than the "onoff" problem (Hauser et al. 1999).

It is probable that the results reflect an understanding of means-end relationships and not a mere discrimination 
Fig. 4 The change in number of correct trials each day (10 trials). a Depicts the number of correct trials of Condition A, b Condition $\mathrm{B}$, $\mathbf{c}$ Condition $\mathrm{C}$, and d Condition $\mathrm{D}$, respectively

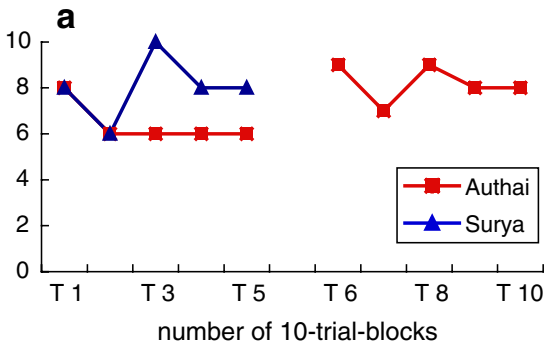

Condition A

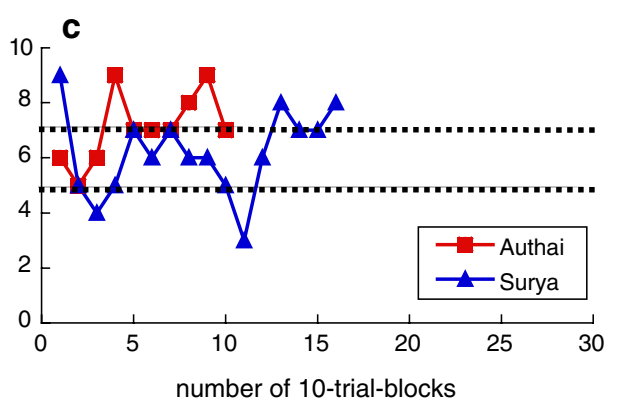

Condition C

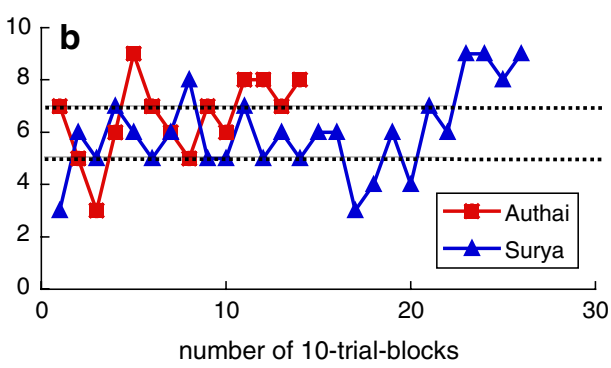

Condition B

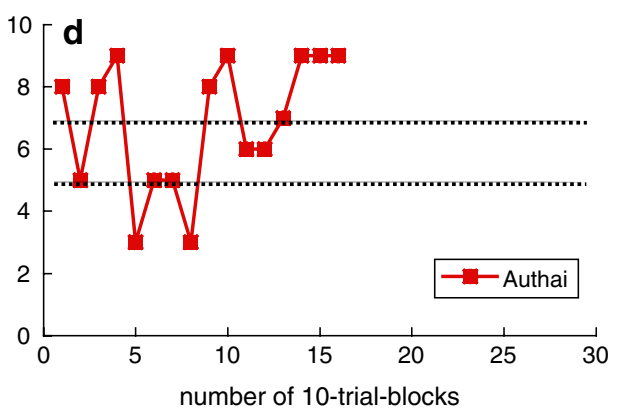

Condition D ability: Authai and Surya were both able to perform above chance level within 50 trials and in contrast, Rensch (1957) reported that the elephant required 330 trials to compare two visual stimuli and choose the correct one. Thus, if our subjects were merely referring to the visual aspect of the stimuli and making a mere visual discrimination, they would have required more trials to complete the tasks.

The elephants' performances were strikingly different from each other. While Authai accomplished all four tasks, Surya was only able to successfully complete the tasks under Conditions A and B. Moreover, in the latter condition, she was much slower than Authai. Considering the fact that food was not deprived this might have resulted in low motivation levels.

Authai's average performance varied between different conditions and blocks. In the first block of Condition A, she would earn the reward regardless of how many times she had pulled a tray within a trial, but in the second block, where she was allowed to choose only once per trial, she had to act correctly to earn the reward. The percentage of correct trials was higher in all the sessions of training two than training one except once.

Authai scored above $66 \%$ in the first blocks of Conditions $\mathrm{C}$ and $\mathrm{D}$, and she was correct in six trials out of her first ten trials for Condition $\mathrm{C}$ and eight trials for Condition D. This suggests that transfer of learning occurred from Condition B. Moreover, her score did not drop during the shift from Condition $\mathrm{B}$ to $\mathrm{C}$, indicating that she understood that the color and size of the tray made no difference to solving the problem. In addition, her score did not drop during the shift from Condition $\mathrm{C}$ to $\mathrm{D}$. The similar performance in Conditions C and D suggests that either the different difficulty levels of the tasks were not reflected in Authai's performance, or that she considered the "on-off" task and the "connectedness" tasks to be identical. Perhaps she considered Condition D as another "onoff" task. When she was shown the trays in the "connectedness" task (Condition D), after she had learnt the "on-off" rule in Conditions B and $\mathrm{C}$, she only looked at those trays that she could draw closer with her trunk.

Thus, there were two different negative stimuli in the experiment: one was the tray on which the food was placed but where she was unable to grab it (Condition D), and the second one was a tray with no food at all (Conditions B and C). The positive stimulus in all conditions was of course the tray containing the bait. Thus, in each task she compared the tray without available food (the negative stimulus) with the positive stimulus. Using this rule, Authai may have solved the "on-off" problem. It is true that Condition D was more complicated than the previous conditions, as she had to know which of the two trays could be drawn closer. However, the rule necessary to solve the problem was the same as that for Conditions B and C, namely the "on-off" rule. Further experiments are necessary to investigate whether such transfer of learning actually occurred and future experiments will require a higher number of subjects if firm conclusions are to be drawn.

Overall, the elephants' performance was rather poor compared to that of nonhuman primates; chimpanzees are able to solve this kind of tasks spontaneously (Povinelli et al. 2000) and tamarins are able to learn the solution more rapidly than do elephants and their performances clearly 
show that their learning was transferred between different types of tools (Hauser et al. 1999, 2002). Certainly, to draw conclusion of the elephants' ability of means-end behavior, experiments with more subjects and more systematically varied conditions need to be done. Also, controlling subjects' motivation levels is another important problem to be solved. The performances of our subjects were not as good as the nonhuman primates of the previous studies. From our results, our subjects seem to be able to solve the means-end task by empirical learning and not spontaneously. However, we found it difficult to keep the elephants concentrated to the task, which might have affected their performance and failed to monitor the accurate learning speed of our subjects. In addition, their limited vision must be taken into account in future studies. Their visual acuity may not be as good as that of primates, although this issue also remains to be addressed.

However, with limitations, this study provides important information that would hint the researchers who plan to run experiments with elephants. Since the study by Rensch (1957), almost no attempt was made to study captive elephants under controlled environments except for the few studies on their mirror self recognition (Povinelli 1989; Plotnik et al. 2006) and some limitedly controlled experiments by Nissani and his colleagues (Nissani et al. 2005; Nissani 2006). This study has shown that elephants are suitable subjects to directly compare their cognition and intelligence with nonhuman primates, and that likely in various cognitive tasks.

Acknowledgements We would like to thank the Ueno Zoological Gardens for their cooperation and support to this study. We thank the ClearScript and Dr. Takafumi Ishida for their editorial support. These experiments comply with the current laws of Japan and the animals are kept at the standards established by Japanese Association of Zoos and Aquariums.

Open Access This article is distributed under the terms of the Creative Commons Attribution Noncommercial License which permits any noncommercial use, distribution, and reproduction in any medium, provided the original author(s) and source are credited.

\section{References}

Gould JL, Gould CG (1994) The animal mind. Scientific American Library, New York

Halsey LG, Bezerra BM, Souto AS (2006) Can wild common marmosets (Callithrix jacchus) solve the parallel strings task? Anim Cogn 9:229-233

Hart BL Hart LA (1994) Fly switching by Asian elephants: tool use to control parasites. Anim Behav 48:35-45
Hart BL, Hart LA, McCoy M, Sarath CR (2001) Cognitive behaviour in Asian elephants: use and modification of branches for fly switching. Anim Behav 62:839-847

Hart BL, Hart LA, Printer-Wollman N (2007) Large brains and cognition: where do elephants fit in? Neurosci Biobehav Rev 32:86-98

Hauser MD, Kralik J, Botto-Mahan C (1999) Problem solving and functional design features: experiments on cotton-top tamarins, Saguinus oedipus oedipus. Anim Behav 57:565-582

Hauser MD, Santos LR, Spaepen GM, Pearson HE (2002) Problem solving, inhibition and domain-specific experience: experiments on cotton-top tamarins. Saguinus Oedipus. Anim Behav 64:387396

Heinrich B, Bugnyar T (2005) Testing problem solving in ravens: string-pulling to reach food. Ethology 111:962-976

Jerison HJ (1974) Evolution of the brain and intelligence. Academic Press, New York

McComb K, Moss C, Syialel S, Baker L (2000) Unusually extensive networks of vocal recognition in African elephants. Anim Behav 59:1103-1109

Nissani M (2006) Do Asian Elephants (Elephas maximus) apply causal reasoning to tool-use tasks? J Exp Psychol Anim Behav Process 32:91-96

Nissani M, Hoefler-Nissani D, Lay UT, Htun UW (2005) Simultaneous visual discrimination in Asian Elephants. J Exp Anal Behav $83: 15-29$

Osthaus B, Lea SEG, Slater AM (2005) Dogs (Canis lupus familiaris) fail to show understanding of means-end connections in a stringpulling task. Anim Cogn 8:37-47

Piaget J (1952) The origins of intelligence in children, (trans. M. Worden). International Universities Press, New York, pp 279-305

Plotnik JM, de Waal FBM, Reiss D (2006) Self-recognition in an Asian elephant. PNAS 103(45):17053-17057

Povinelli DJ (1989) Failure to find self-recognition in Asian elephants (Elephas maximus) in contrast to their use of mirror cues to discover hidden food. J Comp Psychol 103:122-131

Povinelli DJ, Reaux JE, Theali LA, Giambrone S (2000) The support problem: physical connection revisited. In: Povinelli DJ (ed) Folk physics for apes: the chimpanzee's theory of how the world works. Oxford University Press, New York, pp 254-270

Rensch B (1956) Increase of learning capability with increase of brainsize. Am Nat 90:81-95

Rensch B (1957) The intelligence of elephants. Sci Am 196:44-49

Santos LR, Rosati A, Sproul C, Spaulding B, Hauser MD (2005) Means-means-end tool choice in cotton-top tamarins (Sagnuinus Oedipus): finding limits on primates' knowledge of tools. Anim Cogn 8:236-246

Schmidt GF, Cook RG (2006) Mind the gap: means-end discrimination by pigeons. Anim Behav 71:599-608

Vauclair J (1996) Animal cognition: an introduction to modern comparative psychology. Harvard University Press, Cambridge

Willatts P (1984) The stage-IV infant's solution of problems requiring the use of supports. Infant Behav Dev 7:125-134

Willatts P (1990) Development of problem-solving strategies in infancy. In: Bjorklund DF (ed) Children's strategies: contemporary views of cognitive development. Lawrence Erlbaum, Hillsdale, pp 143-182

Willatts P (1999) Development of means-end behavior in young infants: pulling a support to retrieve a distant object. Dev Psychol 35:651-667 